\title{
Custo econômico do absenteísmo de consultas: estudo de caso em um ambulatório de atenção psicossocial
}

\section{Economic cost of consultation absenteeism: case study in a psychosocial care ambulatory Costo económico del absentismo de consulta: estudio de caso em um ambulatorio de atención psicosocial}

Recebido: 29/04/2020 | Revisado: 09/05/2020 | Aceito: 11/05/2020 | Publicado: 20/05/2020

\section{Fabricio Varoni de Oliveira}

ORCID: http://orcid.org/0000-0002-2756-9251

Universidade Federal de Santa Maria, Brasil

E-mail: fvaroni@gmail.com

Reisoli Bender Filho

ORCID: http://orcid.org/0000-0002-1019-4414

Universidade Federal de Santa Maria, Brasil

E-mail: reisolibender@yahoo.com.br

Kelmara Mendes Vieira

ORCID: http://orcid.org/0000-0002-8847-0941

Universidade Federal de Santa Maria, Brasil

E-mail: kelmara@terra.com.br

\section{Resumo}

O absenteísmo ambulatorial pode ser conceituado como a ausência do usuário à consulta e/ou exame previamente agendado ou quando a instituição de saúde é comunicada, no entanto sem possibilitar a comunicação de um novo usuário à consulta e/ou exame não realizado. Cenário este que fundamentou o objetivo de mensurar o custo econômico com o absenteísmo ambulatorial de pacientes do Ambulatório de Atenção Psicossocial do Hospital Universitário de Santa Maria (AAPS/HUSM), no período 2015-2017. Este propósito foi atendido pela utilização do método de custo por absorção. Complementarmente, foram realizadas entrevistas com os agentes participantes do processo de agendamento e execução das consultas com o intuito de obter a percepção destes acerca do absenteísmo ambulatorial e suas repercussões econômicas. Em termos de resultados, a partir da alocação destes custos, obteve- 
se o quantitativo referente às perdas econômicas do absenteísmo que, em média, foi de $\mathrm{R} \$ 396.000,00$ ao ano no triênio analisado para o AAPS/HUSM. No tocante ao custo unitário por consulta, os resultados evidenciaram que o custo médio por paciente agendado foi de $\mathrm{R} \$ 202,92$ enquanto o custo médio por paciente atendido foi de $\mathrm{R} \$ 252,95$. Como determinantes do absenteísmo, evidenciaram-se principalmente aqueles relacionados aos usuários, com destaque ao esquecimento, a falta de consciência e ao descaso do usuário. Quanto ao controle do absenteísmo como suas repercussões nas receitas da instituição, embora o acompanhamento esteja sendo realizado por meio de relatórios, o nível informacional é assimétrico entre os agentes envolvidos no processo de agendamento e execução.

Palavras-chave: Absenteísmo; Custo econômico; Ambulatório de atenção psicossocial; HUSM.

\begin{abstract}
Outpatient absenteeism can be conceptualized as the absence of the user to the appointment and/or examination previously scheduled or when the health institution is communicated, however without allowing the communication of a new user to the consultation and/or examination not performed. This scenario supported the objective of measuring the economic cost of outpatient absenteeism of patients from the Psychosocial Care Outpatient Clinic of the University Hospital of Santa Maria (AAPS/HUSM), in the period 2015-2017. This purpose was met by using the cost per absorption method. In addition, interviews were conducted with agents participating in the appointment scheduling and execution process in order to obtain their perception of outpatient absenteeism and its economic repercussions. In terms of results, from the allocation of these costs, we obtained the amount related to the economic losses of absenteeism, which, on average, was $\mathrm{R} \$ 396.000,00$ per year in the three years analyzed for the AAPS/HUSM. Regarding the unit cost per consultation, the results showed that the average cost per scheduled patient was $\mathrm{R} \$ 202.92$ while the average cost per patient attended was $\mathrm{R} \$ 252.95$. As determinants of absenteeism, it was evidenced mainly those related to users, especially forgetfulness, lack of awareness and neglect of the user. As for the control of absenteeism as its repercussions on the institution's revenues, although the monitoring is being performed through reports, the informational level is asymmetric among the agents involved in the scheduling and execution process.
\end{abstract}

Keywords: Absenteeism; Economic cost; Outpatient psychosocial care; HUSM. 


\section{Resumen}

El ausentismo ambulatorio puede conceptualizarse como la ausencia del usuario a la cita y/o examen previamente programado o cuando se comunica la institución de salud, sin permitir la comunicación de un nuevo usuario a la consulta y/o examen no realizado. Este escenario apoyó el objetivo de medir el costo económico del absentismo ambulatorio de pacientes de la Clínica de atención ambulatoria de atención psicosocial del Hospital Universitario de Santa María (AAPS/HUSM), en el período 2015-2017. Este propósito se cumplió utilizando el método de costo por absorción. Además, se realizaron entrevistas con agentes que participan en el proceso de programación y ejecución de citas para obtener su percepción del absentismo ambulatorio y sus repercusiones económicas. En términos de resultados, a partir de la asignación de estos costos, obtuvimos el monto relacionado con las pérdidas económicas del absentismo, que, en promedio, fue de $\mathrm{R} \$ 396.000,00$ por año en los tres años analizados para el AAPS/HUSM. Con respecto al costo unitario por consulta, los resultados mostraron que el costo promedio por paciente programado fue de $\mathrm{R} \$ 202,92$, mientras que el costo promedio por paciente atendido fue de $\mathrm{R} \$ 252,95$. Como determinantes del absentismo, se evidenciaron principalmente aquellos relacionados con los usuarios, especialmente el olvido, la falta de conciencia y el abandono del usuario. Con respecto al control del absentismo como sus repercusiones en los ingresos de la institución, aunque el monitoreo se realiza a través de informes, el nivel de información es asimétrico entre los agentes involucrados en el proceso de programación y ejecución.

Palabras clave: Absentismo; Costo económico; Ambulatorio de atención psicossocial; HUSM.

\section{Introdução}

A universalização do acesso à saúde, instituída por meio da Constituição Federal de 1988 compõe um dos princípios básicos do Sistema Único de Saúde (SUS). Tal princípio, parte do reconhecimento de que a saúde é direito de todos e um dever do Estado, sendo que este deve garantir o acesso aos bens e serviços sem limitação ou impedimento (Sousa, 2014).

Neste contexto, independentemente do modelo de financiamento, os gastos com saúde tornaram-se fonte de preocupações, não só por representarem parcela significativa do Produto Interno Bruto, como também em virtude de os custos estarem aumentando, muitas vezes com incrementos superiores às taxas econômicas (Piola, Servo, Sá \& Paiva, 2013). Ao mesmo tempo, o desempenho da economia brasileira vem piorando e provocando alterações na 
dinâmica da rede de serviços públicos de saúde, que teve sua demanda aumentada por cidadãos antes beneficiários de planos de saúde. Esta situação tenciona de forma excessiva a rede de saúde pública, marcada, principalmente nos níveis de especialidade, como ponto crítico de estrangulamento no fluxo de pacientes.

Neste contexto, o SUS convive com uma grande pressão de demanda por estes recursos assistenciais, gerando longas filas de espera para alguns procedimentos (Franco \& Magalhães, 2004). Enquanto isso, no âmbito hospitalar, inúmeras instituições desconhecem os custos incorridos em suas atividades por não utilizarem ou até mesmo não disporem de informações geradas pelos sistemas internos para subsidiar as decisões administrativas, o controle de atividades, bem como os investimentos (Falk, 2001; Leoncine, Bornia \& Abbas, 2013).

Embora a apuração dos custos no âmbito da saúde pública seja de grande importância, o debate acerca de medidas que visem aumentar a eficiência do gasto, a fim de mudar a dinâmica das tradicionais modalidades de gestão e prestação de serviços, deve também ser considerada (Organização Pan-Americana da Saúde, 2017). Assim, a ociosidade e suas implicações na rede de saúde devem ser igualmente contempladas, uma vez que o acesso universal à saúde não pode se resumir unicamente à lógica de expansão do sistema (Tristão, 2016).

Nesta linha, o absenteísmo dos usuários nos serviços de saúde, têm sido pauta recorrente nos debates. Embora termo o termo absenteísmo seja frequentemente utilizado à ausência dos profissionais em seu ambiente de trabalho, observa-se sua aplicação também ao fenômeno das faltas dos usuários a compromissos previamente agendados nos serviços de saúde (Oleskovicz, Oliva, Grisi, Lima \& Custódio, 2014). Neste caso, fato de o paciente não comunicar à instituição de saúde a impossibilidade de sua presença à consulta previamente agendada tem impacto relevante em sistemas de saúde onde não há mecanismos reguladores de demanda, dado que permite solicitações ilimitadas de novas consultas (Jabalera Mesa, Morales Asencio \& Rivas Ruiz, 2015).

Assim, o absenteísmo gera um custo econômico, dado que as instituições de saúde estão sujeitas a custos que incorrem independentemente da oferta de serviços. Neste sentido o absenteísmo vai de encontro à sustentabilidade financeira das instituições de saúde, uma vez que as mesmas definem seus processos assistenciais e gerenciais com a finalidade de equilibrar recursos e ações, além de eliminar desperdícios (Castro \& Castilho, 2013). Desta forma, a mensuração das perdas econômicas por desperdício de ociosidade configura-se como uma ação estratégica, sobretudo em estabelecimentos públicos voltados para o atendimento de 
usuários do SUS, os quais convivem com a contradição lógica da obrigação de atender muitos usuários ao mesmo tempo em que possuem espaços com capacidade produtiva real muito abaixo do recomendado e do necessitado (Tristão, 2016).

Pautado neste cenário, de crescimento da demanda por serviços públicos de saúde, em um contexto de escassos recursos que se contrapõe aos gastos decorrentes da não utilização adequada dos serviços de saúde disponíveis, este estudo busca quantificar o custo econômico do absenteísmo de pacientes com consultas agendadas no Ambulatório de Atenção Psicossocial (AAPS) do Hospital Universitário de Santa Maria (HUSM), no período 20152017.

Apesar dos avanços do SUS, por meio da implantação da Estratégia de Saúde da Família e da ampliação do acesso aos serviços de saúde, persiste ainda uma série de desafios a serem enfrentados pelos gestores públicos, materializados por meio da crescente demanda por consultas, exames, medicamentos, cirurgias e mais recentemente judiciais (Ugá, Porto \& Piola, 2012). No entanto, na contramão deste processo, está o volume de recursos que vem se reduzindo, ficando abaixo do necessário para que se cumpra com a responsabilidade de universalização do acesso (Macêdo, Ataide, Costa, Souza \& Santa Rita, 2015).

Assim, a busca pela eficiência na utilização dos recursos é relevante dado o cenário de recursos limitados, sobretudo em instituições que prestam serviços especializados, como os hospitais universitários, os quais possuem custo de produção elevado em virtude da maior densidade de recursos tecnológicos, das atividades de ensino e da complexidade das ações desempenhadas (Bonacim \& Araujo, 2010; Sodré, Littike, Drago \& Perim, 2013). Neste sentido, a mensuração do impacto econômico referente a subutilização dos recursos físicos, sobretudo por sua relevância à tomada de decisão e na gestão racional dos recursos, é um instrumento importante na busca por eficiência.

Dentre os inúmeros desafios ao alcance da eficiência, o absenteísmo revela-se como mais um entrave à produtividade dos serviços ambulatoriais especializados, gerando dificuldade ao acesso de outros usuários aos cuidados de saúde, com reflexos diretos na geração de espaços subutilizados (Bittar, Magalhães, Martines, Felizola \& Falcão, 2016). Situação que tem evidenciado resultados relevantes, mesmo considerando países desenvolvidos, como encontrado nos estudos de (Stone, Palmer, Saxby \& Devaraj, 1999) e (Fysh, 2002), para o Reino Unido e, de (Guerreiro \& Gorgemans, 2009) e (Jabalera Mesa, Morales Asencio, Rivas Ruiz \& Porras González, 2017), à Espanha. No Brasil, os estudos têm abordado esse problema sob o ponto de vista dos motivos pelas quais os pacientes não comparecem às consultas agendadas (Bittar, Magalhães, Martines, Felizola \& Falcão, 2016; 
Mazza \& Costa, 2014; Tristão, 2016; J. S. Santos, 2008) e seus reflexos na atenção básica (Bender, Molina \& Mello, 2010) ou alternativas para redução (Canelada, Levorato, Corte \& Diniz, 2014) e não sob a ótica do custo envolvido.

Além dessa introdução, o estudo compõem-se de outras três seções. Na segunda apresenta-se o processo metodológico, discutindo-se a delimitação e o método de cálculo do absenteísmo. Na terceira discutem-se os resultados, enfatizando o custo do absenteísmo no AAPS/HUSM. E na quarta, delineiam-se as principais considerações sobre o estudo.

\section{Metodologia}

\subsection{Delimitação da pesquisa}

As pesquisas buscam trazer novos saberes para a sociedade como preconiza Pereira et al. (2018) e para tanto faz-se o emprego de metodologias que permitam desvelar conhecimentos que estavam ocultos. $\mathrm{O}$ presente estudo trata-se de uma pesquisa aplicada, com delineamento transversal retrospectivo, com vistas a mensurar o impacto econômico oriundo da ausência dos usuários nas consultas do Ambulatório de Atenção Psicossocial do Hospital Universitário de Santa Maria. Quanto à abordagem, o estudo caracteriza-se como quantitativo, uma vez que são utilizadas informações coletadas de dados institucionais às análises estatísticas.

No que se refere ao objetivo proposto, o estudo classifica-se como descritivo. Para Gil (2002), esse tipo de pesquisa tem como principal característica a descrição das características de determinada população ou fenômeno ou mesmo o estabelecimento de relação entre um conjunto de variáveis. Quanto aos procedimentos técnicos, o estudo insere-se no contexto de um estudo de caso que, conforme Martins (2008) pode ser aplicada quando se deseja avaliar ou descrever situações dinâmicas em que o fator humano está presente.

O caso em estudo é o HUSM, órgão suplementar da Universidade Federal de Santa Maria desde 1970, atua como hospital de ensino, prestando serviços de assistência à saúde integralmente a usuários do SUS, além do desenvolvimento de atividades de ensino. A partir de 17 de dezembro de 2013, a gestão do complexo de saúde passou a ser de responsabilidade da Empresa Brasileira de Serviços Hospitalares (EBSERH). Em termos de estrutura, o hospital movimenta diariamente cerca de 6.000 pessoas, entre pacientes, acompanhantes, alunos, funcionários, residentes e docentes (HUSM, 2017). 
A importância do HUSM no âmbito da saúde pública reflete-se no quantitativo de serviços prestados à população. Quanto à estrutura disponível para serviços que demandam a internação dos usuários, o HUSM conta com 403 leitos, distribuídos em leitos Cirúrgicos, Clínicos, Obstétricos, Pediátricos, Hospital Dia (Saúde Mental e portadores de HIV), para pacientes crônicos e Complementares (Unidades de Cuidados Intermediários, Unidade de Terapia Intensiva).

Os serviços ambulatoriais do hospital são referenciados para os 32 municípios vinculados à $4^{\mathrm{a}}$ Coordenadoria Regional de Saúde (CRS), com sede em Santa Maria e, em algumas especialidades, para os 11 municípios sob jurisdição da $10^{\mathrm{a}} \mathrm{CRS}$, com sede em Alegrete (HUSM, 2017). As ações e serviços de saúde prestados na média e na alta complexidades são referência, atingindo a Macrorregião de Saúde Centro-Oeste do Rio Grande do Sul, composta por três Regiões de Saúde (Verdes Campos, Entre-Rios e Fronteira Oeste), totalizando 1.039.343 habitantes (RIO GRANDE DO SUL, 2016).

Considerando as 15 especialidades que mais agendaram consultas e exames no período de 2015 a 2017, chega-se ao total de 419.859 agendamentos efetuados, representando 68,5\% do total de consultas agendadas (612.878) pela instituição. Além disso, a análise das faltas ambulatoriais sob a perspectiva da especialidade e do tipo agendamento das consultas, revela que, entre as 15 especialidades que mais agendaram consultas, o menor índice refere-se à especialidade Cancerologia $(6,92 \%)$ e a maior índice diz respeito à especialidade Psiquiátrica $(21,21 \%)$, conforme Tabela 1. 
Tabela 1 - Panorama das consultas agendadas dentre as 15 especialidades com maior número de agendamento, entre 2015 e 2017.

\begin{tabular}{lccccc}
\hline Especialidade & $\begin{array}{c}\text { Paciente } \\
\text { Atendido }\end{array}$ & $\begin{array}{c}\text { Paciente } \\
\text { Faltou }\end{array}$ & $\begin{array}{c}\text { Consultas } \\
\text { Agendadas }\end{array}$ & \% Absenteísmo & $\begin{array}{c}\% \\
\text { Presença }\end{array}$ \\
\hline Cancerologia & 54.725 & 4.093 & 59.131 & $6,92 \%$ & $92,55 \%$ \\
Pediatria & 37.091 & 5.405 & 42.876 & $12,61 \%$ & $86,51 \%$ \\
Ginecologia e Obstetrícia & 30.395 & 5.437 & 36.216 & $15,01 \%$ & $83,93 \%$ \\
Enfermagem & 32.609 & 2.665 & 35.482 & $7,51 \%$ & $91,90 \%$ \\
Fonoaudiologia & 25.341 & 3.450 & 29.259 & $11,79 \%$ & $86,61 \%$ \\
Psiquiatria & 21.531 & 5.846 & 27.560 & $21,21 \%$ & $78,12 \%$ \\
Endocrinologia e Metabologia & 23.143 & 3.574 & 27.101 & $13,19 \%$ & $85,40 \%$ \\
Dermatologia & 21.222 & 4.135 & 25.452 & $16,25 \%$ & $83,38 \%$ \\
Ortopedia e Traumatologia & 21.472 & 3.282 & 25.247 & $13,00 \%$ & $85,05 \%$ \\
Gastroenterologia & 20.019 & 3.988 & 24.088 & $16,56 \%$ & $83,11 \%$ \\
Urologia & 17.775 & 2.530 & 20.364 & $12,42 \%$ & $87,29 \%$ \\
Otorrinolaringologia & 14.959 & 3.062 & 18.245 & $16,78 \%$ & $81,99 \%$ \\
Reumatologia & 15.330 & 1.831 & 17.642 & $10,38 \%$ & $86,89 \%$ \\
Pneumologia & 14.533 & 2.689 & 17.471 & $15,39 \%$ & $83,18 \%$ \\
Cardiologia & 11.513 & 1.867 & 13.725 & $13,60 \%$ & $83,88 \%$ \\
Demais & 164.243 & 24.578 & 193.019 & $12,73 \%$ & $85,09 \%$ \\
\hline Total & 525.901 & 78.432 & 612.878 & - & - \\
Média (ano) & 175.300 & 26.144 & 204.293 & - & - \\
\hline
\end{tabular}

Fonte: HUSM (2018).

Tendo em vista percepção dos usuários acerca da letalidade dos casos de câncer e do conhecimento de que o diagnóstico tardio dificulta o tratamento, reduzindo o tempo de sobrevida e qualidade de vida do paciente, encontrar baixo índice de faltas neste tipo de especialidade não causa surpresa (Silva et al., 2015).

Por sua vez, quanto ao o índice de $21,21 \%$ de ausências dos pacientes agendados no AAPS, embora menor que os 36\% registrados por Bittar et al. (2016), foi ligeiramente superior ao índice de 20,5\% encontrado por Giacchero \& Miasso (2008), considerando resultados em um ambulatório de psiquiatria do interior do estado de São Paulo. A ausência nesta especialidade pode repercutir na adesão às terapias medicamentosas, muito comum à esta categoria, podendo comprometer as condições clínicas e a qualidade de vida dos pacientes.

Ao desagregar os dados sob a perspectiva do tipo de consulta, os valores sinalizam a Psiquiatria como destaque em todas as análises. Quanto ao índice de ausência em primeiras consultas, nas quais as 15 especialidades que mais agendaram respondem por $71,31 \%$ do total de novas consultas agendadas no período, o menor índice de comparecimento corresponde aos usuários do AAPS, atingindo 72,32\%. 
Este resultado é expressivo, visto que os usuários que não comparecem às primeiras consultas devem retornar aos postos de saúde para conseguir novo referenciamento, pressionando ainda mais o sistema de saúde pública. Conforme argumenta Barcelos (2016), os baixos índices de presença em primeiras consultas podem estar relacionados a falhas de comunicação entre as centrais de regulação e os usuários.

No que concerne às consultas de retorno, às quais correspondem aos encontros de acompanhamento originado a partir de uma primeira consulta, os índices de não comparecimento igualmente são elevados, correspondendo a 20,22\% na Psiquiatria. Esta categoria de consulta é a mais demandada no AAPS, uma vez que o tratamento dos pacientes nesta especialidade, por vezes, desenvolve-se por longos períodos.

O absenteísmo em consultas de retorno da Psiquiatria pode estar associado à inadequação do paciente à conduta, aos resultados do tratamento ou até mesmo barreiras financeiras, podendo ter como consequência o recrudescimento do problema, evoluindo à deterioração do estado de saúde com possiblidade de recorrer à internação (Killaspy, Banerjee, King \& Lloyd, 2000). Logo, o não comparecimento do paciente às consultas podem acarretar um maior dispêndio de recursos públicos, visto que a internação hospitalar possui maior custo quando comparada ao acompanhamento ambulatorial.

Outra modalidade de atendimento ambulatorial, a interconsulta, refere-se à consulta requisitada entre especialidades médicas diferentes e por profissionais cujas atividades desenvolvem-se no próprio HUSM. No período de 2015 a 2017, dentre as 15 especialidades que mais agendaram consultas na instituição, o menor índice de comparecimento nesta modalidade de consulta igualmente está vinculado aos pacientes da AAPS (34,57\%).

Os motivos de tais ausências podem estar relacionados, assim como a primeira consulta, a percepção, por parte do paciente, que o seu problema está resolvido ou com a discordância acerca da necessidade do encaminhamento à consulta psiquiátrica (Killaspy et al., 2000). Ainda, para Pereira-Victorio, Vázquez-Molinero, Lizán-Garcia, Gómez-Guardiola \& López-Valcárcel (2016), a ausência dos usuários nesta modalidade pode estar associada a motivos alheios à sua responsabilidade.

Portanto, em função dos níveis de absenteísmo, tanto nas primeiras consultas quanto na interconsulta, optou-se por escolher o APPS como o setor dentro do HUSM a ser objeto do estudo. E, a escolha de apenas uma especialidade, deveu-se à complexidade de obtenção dos dados para a estimação do custo. 


\subsection{Método de cálculo}

Com o intuito de valorar os custos econômicos incorridos em virtude da ausência dos pacientes nos compromissos agendados no AAPS, é utilizado o método de análise de custos por absorção. A utilização do referido método de custeio, justifica-se por ser o único sistema de custeio aceito pela legislação (Lima \& Moraes, 2016), por sua ampla utilização nas instituições de saúde e pelo fato de ser a metodologia utilizada pelo Programa Nacional de Gestão do Custo (PNGC) (Arruda, 2014; Ministério da Saúde \& Organização Pan-Americana da Saúde, 2013).

Neste método, tanto os custos fixos (aqueles que não são afetados pela quantidade produzida) quantos os custos variáveis (aqueles cujo montante é afetado pelo volume produzido) devem ser absorvidos no custo dos bens e serviços produzidos (Vieira, 2017).

No estudo são considerados como custos diretos aqueles relacionados diretamente aos produtos ou serviços de saúde prestados, sendo estes originários do próprio ambulatório: gastos com pessoal (médicos, enfermeiros e residentes). Por sua vez, Siqueira Filha (2011) considera como custos indiretos: atividades administrativas, recepcionistas, serviço de arquivo médico, limpeza, manutenção e suporte em computadores, manutenção e suporte em telefonia, radiocomunicadores, vigilância, monitoramento, energia elétrica e água. As expressões utilizadas aos referidos cálculos encontram-se no Quadro 1. 
Quadro 1 - Classificação dos custos do AAPS e fórmulas de cálculo.

\begin{tabular}{|c|c|c|}
\hline \multicolumn{3}{|r|}{ CUSTOS DIRETOS } \\
\hline \multicolumn{2}{|l|}{ Itens } & Fórmula de Cálculo \\
\hline \multicolumn{2}{|l|}{ Médicos } & $\sum$ Salário e encargos sociais \\
\hline \multicolumn{2}{|l|}{ Enfermeiros } & $\sum$ Salário e encargos sociais \\
\hline \multicolumn{2}{|l|}{ Residentes } & $\sum$ Bolsas de estudo \\
\hline \multicolumn{3}{|r|}{ CUSTOS INDIRETOS } \\
\hline \multicolumn{2}{|l|}{ Itens } & Fórmula de Cálculo \\
\hline \multicolumn{2}{|l|}{ Administrativo (AAPS) } & $\begin{array}{l}\sum \text { Salário e encargos sociais }+\sum \text { Salário e encargos sociais do chefe da } \\
\text { unidade/2 }\end{array}$ \\
\hline \multicolumn{2}{|l|}{ Recepcionistas (AAPS) } & $\sum$ Custo contratualizado $\mathrm{x} F$ \\
\hline \multicolumn{2}{|c|}{ Serviço de Arquivo Médico } & $\sum$ Custo do Serviço de Arquivo Médico/movimentação prontuários \\
\hline \multicolumn{2}{|l|}{ Serviço de Limpeza } & (Valor cobrado por m2 x Área AAPS) \\
\hline \multicolumn{2}{|c|}{$\begin{array}{l}\text { Manutenção e suporte em } \\
\text { Computadores }\end{array}$} & $\begin{array}{l}\text { ( } \sum \text { Custo do SGPTIcomputadores } / \mathrm{n}^{\circ} \text { de computadores) } \mathrm{x} \text { total de } \\
\text { computadores no AAPS }\end{array}$ \\
\hline \multicolumn{2}{|c|}{ Manutenção e suporte em Telefonia } & $\begin{array}{l}\text { ( } \sum \text { Custo do SGPTItelefonia } / \mathrm{n}^{\circ} \text { de ramais) } \mathrm{x} \text { total de ramais no } \\
\text { AAPS }\end{array}$ \\
\hline \multicolumn{2}{|l|}{ Ligações telefônicas } & Custo dos ramais telefônicos AASP \\
\hline Itens & & Fórmula de Cálculo \\
\hline \multicolumn{2}{|l|}{ Radiocomunicadores } & tualizado $/ n^{\circ}$ de radiocomunicadores) x 2] x F \\
\hline \multicolumn{2}{|l|}{ Vigilância } & o de trabalho $\mathrm{x} F$ \\
\hline Monitoramento & $\begin{array}{l}{[(\text { Custo con }} \\
\text { AAPS }] \times \text { F }\end{array}$ & tualizado / Pontos de Monitoramento) x Pontos de monitoramento \\
\hline Água & (Custo com & viço de Água/área AAPS) x F \\
\hline Energia Elétrica & (Custo com & erviço de Energia Elétrica/área AAPS) x F \\
\hline
\end{tabular}

Fonte: Elaborado pelos autores baseado em Brittes (2016).

Nota: F representa a fração de consultas agendadas pela especialidade Psiquiatria em relação ao total de consultas ofertadas no AAPS (Psiquiatria + Neurologia).

Ressalta-se que, para o custo do profissional (custos diretos) foram identificadas as categorias profissionais relacionadas direta ou indiretamente à prestação de serviço que correspondem aos salários e aos encargos sociais dos profissionais. Estes valores serão coletados junto ao Portal de Transparência do Governo Federal15, no caso dos profissionais vinculados ao Regime Jurídico Único; para os profissionais vinculados ao Regime Geral de Previdência, os dados foram no Portal de Transparência da EBSERH; quanto aos profissionais residentes, as informações tiveram como fonte as Portarias Interministeriais que tratam das Residência Médica. E os valores para os serviços terceirizados, como segurança, monitoramento, limpeza e recepcionistas, (custos indiretos), foram coletados junto à Unidade de Contratos do Hospital Universitário de Santa Maria.

Ainda, posto que no espaço ambulatorial são ofertadas consultas médicas da especialidade Neurologia, o custo oriundo das atividades de recepção, radiocomunicadores, 
(CC BY 4.0) | ISSN 2525-3409 | DOI: http://dx.doi.org/10.33448/rsd-v9i7.4066

vigilância monitoramento, água e energia elétrica foram rateados tomando como base a porcentagem representativa de cada especialidade no quantitativo de consultas agendadas na unidade. Dessa forma, torna-se possível dividir os custos entre as especialidades que lá desempenham suas atividades, evitando, assim, a distorção dos resultados.

À consecução do objetivo, foram acessadas fontes de dados a partir de setores da própria instituição e fontes externas. Dentre estas, citam-se os Portais de Transparência do Governo Federal e da EBSERH, para ter acesso aos salários dos profissionais não terceirizados, e Portarias Interministeriais, que apresentam o valor pago em bolsa de residência. O período utilizado engloba os anos de 2015 a 2017, sendo os resultados calculados anualmente

\section{Resultados e Discussão}

\subsection{Consultas e absenteísmo no AAPS/HUSM}

O quantitativo de consultas médicas comportou-se de maneira irregular no período analisado. Em 2015, o quantitativo foi de 7.121, aumentando para 10.630 em 2016 e reduzindo-se para 9.809 em 2017. Por outro lado, as consultas de enfermagem apresentaram retração de cerca de 30\% no período, passando de 1.075 em 2015 para 752 em 2017.

Quanto aos percentuais de absenteísmo, houve diminuição gradual no período para consultas médicas, que em 2015 representava 23,5\% e em 2017 atingiu 19,8\%, enquanto as consultas de enfermagem apresentaram baixos índices de ausências, com exceção do ano 2016 que foi de 12\%. Assim, torna-se possível verificar que as consultas médicas apresentaram o maior volume de consultas agendadas e também os maiores índices de absenteísmo, apesar da diminuição média de (-8,21\%) ao ano entre 2015 e 2017.

No que diz respeito à média de idade, houve elevação gradual entre 2015 e 2016 (Tabela 2), sendo que a média de idade dos usuários faltantes sempre foi inferior à média de idade dos usuários que compareciam. 
Research, Society and Development, v. 9, n. 7, e417974066, 2020

(CC BY 4.0) | ISSN 2525-3409 | DOI: http://dx.doi.org/10.33448/rsd-v9i7.4066

Tabela 2 - Média de idade dos usuários atendidos e não atendidos.

\begin{tabular}{lccc}
\hline & 2015 & 2016 & 2017 \\
\hline Usuário atendido & 43,7 & 45,1 & 44,7 \\
Usuário faltou & 38,8 & 41,9 & 42,3 \\
\hline \hline & 2015 & 2016 & 2017 \\
\hline$t$ & $-9,2$ & $-8,5$ & $-5,7$ \\
Sig. & 0,000 & 0,000 & 0,000 \\
\hline
\end{tabular}

Fonte: Resultado da pesquisa. Elaborado pelos autores.

Estatisticamente houve diferença para os três anos (Sig. 0,000), evidenciando que os pacientes mais jovens tendem a comparecer menos. Esta situação pode estar relacionada com o fato de esta população estar classificada como população economicamente ativa, como reportado em estudo de Izecksohn \& Ferreira (2014).

O tempo entre o agendamento da consulta e a sua realização mostrou que houve aumento no número de dias à efetiva consulta tanto para a categoria de usuários atendidos quanto para usuários que faltaram. Contudo, a média de dias dos usuários que faltaram foi superior à média de dias dos pacientes que comparecerem, sendo as diferenças entre as médias estatisticamente diferentes o período de análise.

Tabela 3 - Média de dias entre marcação/consulta.

\begin{tabular}{lccc}
\hline & 2015 & 2016 & 2017 \\
\hline Usuário atendido & 15,0 & 13,1 & 20,8 \\
Usuário faltou & 18,9 & 14,7 & 22,5 \\
\hline & 2015 & 2016 & 2017 \\
\hline$t$ & 5,098 & 4,001 & 4,154 \\
Sig. & 0,000 & 0,000 & 0,000 \\
\hline
\end{tabular}

Fonte: Resultado da pesquisa. Elaborado pelos autores.

Do ponto de vista do volume de agendamentos ao longo da semana, verificou-se concentração entre terças e quintas-feiras. Por outro lado, o absenteísmo eleva-se na segundafeira e decresce ao longo da semana, até quinta-feira, quando volta a subir. Quanto a estes aspectos, Kheirkhash, Feng, Travis, Tavakoli-Tabasi \& Sharafkhaneh (2016) mostraram que o percentual de ausência em consultas, assim como encontrado, é superior na segunda-feira quando comparado com os demais dias da semana. Para Ellis \& Jenkins (2012), o menor índice de presenças nas segundas-feiras pode estar ligado às associações emocionalmente negativas com a segunda-feira feitas pelos usuários. 
Quanto ao horário em que as consultas ocorrem, segundo dados da AGHU, estão fundamentalmente concentradas às 13 horas, com diminuição progressiva até às 18 horas. Já o absenteísmo é elevado nas consultas agendadas às 7 horas, mas diminui progressivamente até às 11 horas, quando aumenta e permanece na faixa dos $20 \%$, aumentando para $31 \%$ nos horários finais de expediente, sobremaneira a partir das 16 horas.

Complementando, a distribuição das consultas do AAPS/HUSM mostra que os agendamentos se dão fundamentalmente para os usuários de Santa Maria (66,06\%), contudo não é o município que apresenta o maior absenteísmo. Dentre os 15 municípios que mais agendaram consultas no período analisado, seis tiveram seus índices de faltas superiores à $20 \%$, com destaque para São Pedro do Sul, que apresentou o maior $(24,7 \%)$, seguido de Júlio de Castilhos $(23,9 \%)$.

\subsection{Análise do custo do absenteísmo}

Considerando a totalidade dos recursos, o custo do AAPS/HUSM superou R $\$ 2$ milhões ao ano no período analisado (Ver APÊNDICE A). A maior parcela de custos anual decorre da folha de pagamento, que foi responsável por cerca de $72 \%$ do total, com destaque para o pagamento dos profissionais médicos, que representou $60,7 \%$, em média, do volume de custos da unidade.

Para perfazer o custo com os salários dos profissionais médicos, foi utilizada a remuneração básica bruta (RBB) extraída do Portal de Transparência do Governo Federal e do Portal de Transparência da EBSERH. Porém, como o esse último disponibiliza apenas as seis últimas folhas de pagamento, projetou-se para os demais meses a RBB de dezembro de 2018 . Ainda, cabe destacar que, apesar de alguns médicos possuírem vínculo assistencial e docente, suas remunerações foram consideradas de forma integral. Para compor o custo total com bolsas dos residentes, utilizou-se da multiplicação do número de residentes de segundo, terceiro e quarto ano pelos valores constantes nas Portarias Interministeriais n. 9/2013 e $3 / 2016$.

À categoria administrativa obteve-se parcela de 6,07\%, em média, do custo total da unidade, com crescimento médio de 10,55\% ao ano (ver APÊNDICE A). Para compor o custo deste item, foi considerado o salário de um servidor acrescido de metade do salário do responsável pela unidade, considerando a hipótese deste desempenhar tarefas administrativas na unidade de internação e ambulatorial. 
Os valores referentes aos itens recepcionistas, vigilância e monitoramento apresentaram comportamento similar, aumentando suas participações no custo final no biênio 2015-2016, com queda no último ano da análise. Por outro lado, o item radiocomunicadores, apesar de variar quantitativamente, apresentou participação constante no custo total do período.

Os dados para compor custos com recepcionistas, vigilância, radiocomunicadores e monitoramento tiveram como fonte os contratos com as empresas terceirizadas. Especificamente para o custo de monitoramento considerou-se o valor por câmera monitorada; aos radiocomunicadores foram considerados dois, sendo um para o serviço de vigilância e outro para a central de monitoramento e, por fim, o serviço de vigilância foi valorado considerando que o posto de vigilância no AAPS é exclusivo da unidade e seu valor estabelecido em contrato com a empresa terceirizada; quanto às recepcionistas, foram consideradas duas no período de análise e o seu custo estabelecido por meio de contrato.

No que se refere aos itens ligações telefônicas, serviço de água e energia elétrica verificou-se comportamento diferenciado. Enquanto a participação média no custo total com energia elétrica foi de $1,27 \%$ no período, a participação com serviço de água teve média de $0,21 \%$ e de apenas $0,02 \%$ o custo com ligações telefônicas.

Para calcular o dispêndio com os referidos itens de energia elétrica e água do AAPS foi utilizado a fração de área da unidade em relação a área total $(0,013386)$ que, multiplicado pelo valor total pago pelo hospital, chegou-se a um valor estimado de consumo da unidade. Para valorar o custo com ligações telefônicas, tomou-se como base o valor gasto no segundo semestre de 2018.

Os serviços de manutenção e suporte em computadores e telefonia tiveram participação inferior a $1 \%$ no custo total da unidade. Quanto ao item manutenção e suporte em telefonia, houve crescente participação nos custos totais, enquanto o item manutenção e suporte de computadores apresentou aumento de 2015 para 2016 e estabilização de 2016 para 2017.

Para os itens de manutenção e suporte em computadores e em telefonia, foram considerados os custos com folha de pagamento, bolsa estudantil, pessoal terceirizado, luz, água e limpeza. Tanto o dispêndio com a luz quanto com a água, foram calculados levando-se em consideração a proporção de área que as unidades ocupam $(0,002244)$ em relação a área total da instituição. O custo com limpeza foi valorado multiplicando-se o custo por $\mathrm{m}^{2}$ contratualizado pela área que as unidades ocupam $\left(87,5 \mathrm{~m}^{2}\right)$. 
O Serviço de Arquivo apresentou participação média de cerca de 1,5\% do custo total da unidade, sendo o ano de 2016 o que apresentou maior volume de recursos, correspondente a $1,70 \%$. Para compor esse custo foi considerada a folha de pagamento dos funcionários efetivos, do custo com o pessoal terceirizado, com bolsista, energia elétrica, água e limpeza. À obtenção do dispêndio com energia elétrica e água deste serviço, multiplicou-se a fração de área do Serviço de Arquivo $(0,00841)$ pelo gasto total da instituição com água e energia elétrica. Para mensurar o gasto com limpeza, multiplicou-se o custo por $\mathrm{m}^{2}$, estabelecido em contrato com a empresa terceirizada, pela área do Serviço de Arquivo $\left(328 \mathrm{~m}^{2}\right)$. Para mensurar o custo repassado para o AAPS, o custo total do Serviço de Arquivo foi dividido pelo número de prontuários movimentados anualmente. De posse de custo/prontuário, multiplicou-se este valor pelo número de consultas agendadas no AAPS.

A partir deste conjunto de informações de custos e, considerando o somatório das categorias e dos itens que formam o AAPS, obteve-se o custo médio por paciente atendido para os anos 2015-2017, que foi de $\mathrm{R} \$ 252,95$ (Tabela 4). Por sua vez, o custo médio com o paciente agendado foi de $\mathrm{R} \$ 202,92$ sendo encontrado o menor valor para o ano de 2016 $(\mathrm{R} \$ 174,47)$.

Tabela 4 - Custo por paciente no AAPS/HUSM entre período de 2015 a 2017, em R\$.

\begin{tabular}{lcccc}
\hline & 2015 & 2016 & 2017 & Média \\
\hline Custo por Paciente Agendado & 230,74 & 174,47 & 203,54 & 202,92 \\
Custo por Paciente Atendido & 290,19 & 219,00 & 249,67 & 252,95 \\
\hline
\end{tabular}

Fonte: Resultado da pesquisa. Elaborada pelos autores.

Quando avaliados comparativamente com outras unidades de saúde, os respectivos valores não distancia-se expressivamente, caso de Brittes (2016) que valorou em $\mathrm{R} \$ 233,32$ o atendimento de emergência para um hospital público da região metropolitana de Porto Alegre. Por outro lado, ao avaliar uma Unidade de Cuidados Integrais de Recife, Carvalho (2016) valorou em R \$110,75 o atendimento individual enquanto Assunção (2016), em João Pessoa, valorou em $\mathrm{R} \$ 110,49$.

A diferença de valores encontrada pode estar relacionada ao fato do AAPS/HUSM ser um espaço que congrega atividades de docência, o que torna sua estrutura mais dispendiosa (Santos, Borgert \& Borgert, 2017). Além disso, em suas dependências é desenvolvido um conjunto de ações, práticas, conhecimentos e técnicas assistenciais com maior densidade tecnológica, o que contribui para explicar o maior custo (Médici, 2001; Solla \& Chioro, 2014). 
Estes quantitativos são centrais ao processo de gestão do custo nas organizações de saúde e permitem a mensuração de perdas. Neste contexto, de posse do custo por paciente agendado no AAPS/HUSM e dos quantitativos de pacientes que não compareceram em consultas no período, foi possível quantificar uma perda média de $\mathrm{R} \$ 396$ mil por ano correspondente ao absenteísmo, sendo 2016 o ano com maior perda e 2015 o de menor (Tabela 5).

Tabela 5 - Custo econômico do absenteísmo no AAPS/HUSM no período de 2015 a 2017, em R\$.

\begin{tabular}{lcccc}
\hline & 2015 & 2016 & 2017 & Média \\
\hline Custo por Paciente agendado & 230,74 & 174,47 & 203,54 & 202,92 \\
Consultas perdidas & 1.679 & 2.324 & 1.951 & 1.985 \\
CEA $^{1}$ & $387.414,92$ & $405.460,64$ & $397.112,40$ & $396.662,66$ \\
\hline
\end{tabular}

Fonte: Resultado da pesquisa. Elaborado pelos autores.

${ }^{1}$ Custo Econômico do Absenteísmo.

Tomando como base a própria matriz de custos da unidade, o que é perdido com absenteísmo, corresponde a um quinto do custo total, superando inclusive o valor pago com bolsas para os nove residentes. Neste sentido, o quantitativo de perdas é expressivo e sinaliza para a necessidade de ações específicas e de curto prazo com o intuito de minimizar as perdas e otimizar os recursos.

Apesar de o ano 2016 ter apresentado o menor custo por paciente, a perda com o absenteísmo foi a maior do período. Desta forma, dado que os custos com o AAPS/HUSM são praticamente constantes, a relação custo/paciente somente pode ser alterada por meio do número de atendimentos. Logo, faz-se necessário que o AAPS/HUSM não somente busque trabalhar próximo da sua capacidade operacional por meio de medidas que ampliem o número de atendimentos realizados, como também analise a possibilidade de incentivar a diminuição do absenteísmo por meio da relação profissional-paciente.

\section{Considerações Finais}

O trabalho avaliou o impacto econômico decorrente do absenteísmo do Ambulatório de Atenção Psicossocial do HUSM, entre os anos de 2015 a 2017. A partir da alocação dos custos, obteve-se o resultado referente às perdas econômicas do absenteísmo que, em média, foi de R\$396 mil ao ano, no triênio analisado, para o AAPS/HUSM. No tocante ao custo por 
consulta, incluindo o custo com infraestrutura, os resultados apontam que o custo médio por paciente atendido foi de $\mathrm{R} \$ 252,95$ e o custo médio com paciente agendado foi de $\mathrm{R} \$ 202,92$. Dentre os itens de custo que mais contribuem no custo total do ambulatório está a folha de pagamento dos servidores médicos, que responde por $60,7 \%$.

O custo médio por paciente atendido demonstra a expressiva distorção entre os custos incorridos e os valores repassados aos prestadores pelo Ministério da Saúde. Enquanto o custo médio por consulta foi de $\mathrm{R} \$ 210,45$, quando considerados somente os custos com folha de pagamento dos profissionais diretamente envolvidos nos atendimentos (médicos, enfermeiros e residentes), o valor repassado são de $\mathrm{R} \$ 10,00$ e $\mathrm{R} \$ 6,30$, para consultas médicas e de enfermagem, se considerada a hipótese de todos os procedimentos estarem contratualizados junto à Secretaria Estadual de Saúde.

A partir desses resultados pode-se concluir que o custo econômico com o absenteísmo no AAPS/HUSM não poder ser desconsiderado, uma vez que supera inclusive o dispêndio com as bolsas de estudos consideradas. Este fato merece a atenção dos gestores da instituição e demonstra a necessidade de maior integração com as demais partes do sistema de saúde (coordenadoria e secretaria) visando não somente mitigar o problema, mas também equacionar as perdas econômicas que pesam sobre o prestador de serviços.

Ainda, os problemas do absenteísmo na instituição são reflexos de condicionantes que colocam à prova sua capacidade de negociação tanto em âmbito interno quanto externo. No externo, a relação com os municípios deve ser priorizada, mantendo sempre um canal à resolução de problemas, como a questão dos transportes; enquanto que, no institucional, os gestores devem trabalhar no sentido de massificar as informações acerca do absenteísmo permitindo que, principalmente os servidores assistenciais, apropriem-se delas e as utilizem na sua interação com os usuários.

Apesar de as análises terem permitido o conhecimento mais detalhado acerca do absenteísmo no AAPS/HUSM, a temática não se esgota em termos de resultados e análises. A utilização de dois sistemas de informação diferenciados (SIE e AGHU) limitou o maior aprofundamento das análises em função da incompatibilidade de parte do banco de dados. A utilização dos dados salariais dos trabalhadores da EBSERH também limitou as análises uma vez que o portal de transparência somente disponibiliza as seis últimas folhas de pagamento, sendo necessário realizar algumas simplificações.

Por fim, propõe-se que seja pesquisado as ausências às consultas sob o ponto de vista do usuário, buscando estruturar o perfil destes pacientes e principalmente as barreiras de acesso a que estão submetidos. Também pesquisar a viabilidade de utilização de contato 
telefônico e de ferramentas de lembrete de consultas e exames como ações de redução do absenteísmo. Ainda, ressalta-se a importância de examinar outras unidades/especialidades para se obter um panorama mais amplo a respeito dos custos e dos determinantes do absenteísmo.

\section{Referências}

Arruda, KG. (2014). Avaliação dos custos do tratamento de tuberculose em município de médio porte do nordeste brasileiro. (Dissertação de Mestrado). Universidade Federal de Pernambuco, Recife, PE.

Assunção, MCT. (2016). Análise de custos de um serviço de práticas integrativas e complementares no município de João Pessoa - Paraíba. (Dissertação de Mestrado). Universidade Federal de Pernambuco, Recife, PE.

Barcelos, GM. (2016). Aproveitamento de vagas de consultas eletivas de um hospital universitário. (Dissertação de Mestrado). Universidade de São Paulo, Rib. Preto, SP.

Bender, AS, Molina, LR \& Mello, ALSF. (2010). Absenteísmo na atenção secundária e suas implicações na atenção básica. Revista Espaço para a Saúde, 11(2), pp. 56-65.

Bittar, O. J. N. V., Magalhães, A., Martines, C. M., Felizola, N. B. G., \& Falcão, L. H. B. (2016). Absenteísmo em atendimento ambulatorial de especialidades no estado de São Paulo. Bepa-Boletim Epidemiológico Paulista, 13(152), pp. 19-32.

Bonacim, CAG \& Araujo, AMP. (2010). Gestão de custos aplicada a hospitais universitários públicos: a experiência do Hospital das Clínicas da Faculdade de Medicina de Ribeirão Preto da USP. Revista de Administração Pública, 44(4), pp. 903-931.

Brittes, MS. (2016). Implantação de gestão de custos no setor de emergência de um hospital público. (Trabalho de Conclusão de Curso). Universidade Federal do Rio Grande do Sul, Porto Alegre, RS. 
Canelada, HF, Levorato, CD, Corte, RIAS \& Diniz, EES. (2014). Redução do absenteísmo através da gestão da agenda e do trabalho em rede. Blucher Medical Proceedings, 1(2), p.145.

Carvalho Neto, MR. (2016). Avaliação dos custos da Unidade de Cuidados Integrais Prof. Guilherme Abath, Recife. (Dissertação de Mestrado). Universidade Federal de Pernambuco, Recife, PE.

Castro, LC \& Castilho, V. (2013). O custo de desperdício de materiais de consumo em um centro cirúrgico. Revista Latino-Americana de Enfermagem, 21(6), pp. 1228-1234.

Ellis, DA \& Jenkins, R. (2012). Weekday affects attendance rate for medical appointments: large-scale data analysis and implications. PloS one, 7(12), p. e51365.

Falk, JA. (2001). Gestão de custos para hospitais: conceitos, metodologias e aplicações. São Paulo, SP: Atlas.

Franco, TB \& Magalhães, HVJr. (2004). Integralidade na assistência à saúde: a organização das linhas de cuidado. In E. E. Merhy (Org) O Trabalho em Saúde: olhando e experienciando o SUS no cotidiano (2 ed). São Paulo, SP: Hucitec.

Fysh, T. (2002). Missed outpatient appointments. Journal of the Royal Society of Medicine, 95(7), pp. 376-377.

Giacchero, KG \& Miasso, AI. (2008). Ambulatório de psiquiatria em hospital geral: caracterização da adesão de usuários ao agendamento. Revista da Rede de Enfermagem do Nordeste, 9(2), pp. 20-27.

Gil, AC. (2002). Como elaborar projetos de pesquisa. 4 ed. São Paulo, SP: Atlas.

Guerrero, MA \& Gorgemans, S. (2009). Absentismo de pacientes citados en las consultas de Atención Especializada del Consorcio Aragonés Sanitario de Alta Resolución: repercusión económica y demoras. In Anais do XVI Encuentro de Economía Pública, Granada, Espanha. 
Hospital Universitário de Santa Maria - HUSM. (2017). Serviço de Estatística/Relatório Estatístico. Santa Maria, RS.

Izecksohn, MMV \& Ferreira, JT. (2014). Falta às consultas médicas agendadas: percepções dos usuários acompanhados pela Estratégia de Saúde da Família, Manguinhos, Rio de Janeiro. Revista Brasileira de Medicina de Família e Comunidade, 9(32), pp. 235-241.

Jabalera Mesa, ML, Morales Asencio, JM \& Rivas Ruiz, F. (2015). Factores determinantes del absentismo en consultas externas de la Agencia Sanitaria Costa del Sol. In Anales Sis San Navarra, Pamplona, Espanha.

Jabalera Mesa, ML, Morales Asencio, JM, Rivas Ruiz, F \& Porras González, MH. (2017). Análisis del coste económico del absentismo de pacientes en consultas externas. Revista de Calidad Asistencial, 32(4), pp. 194-199.

Kheirkhah, P, Feng, Q, Travis, LM, Tavakoli-Tabasi, S \& Sharafkhaneh. A. (2016). Prevalence, predictors and economic consequences of no-shows. BMC Health Services Research, 16(1), p. 13.

Killaspy, H, Banerjee, S \& Lloyd, M. (2000). Prospective controlled study of psychiatric outpatient non-attendance: Characteristics and outcome. The British Journal of Psychiatry, 176(2), pp. 160-165.

Leoncine, M, Bornia, AC \& Abbas, K. (2013). Sistemática para apuração de custos por procedimento médico-hospitalar. Production, 23(3), pp. 595-608.

Lima, FF \& Moraes Filho, RA. (2016). Gestão estratégica de custos: custeio por absorção em pequenas empresas em Recife, PE, Brasil. Interações (Campo Grande), 17(3), pp. 528-541.

Macêdo, DF, Ataide, JAR, Costa, ACS, Souza, WAR \& Santa Rita, LP. (2015). Análise da judicialização do direito à saúde, subfinanciamento do setor e políticas públicas: estudo de caso no estado de Alagoas. Revista de Administração de Roraima, 5(2), pp. 300-325. 
Martins, GA. (2008). Estudo de caso: uma reflexão sobre a aplicabilidade em pesquisas no Brasil. Revista de Contabilidade e Organizações - FEARP/USP, 2(2), pp. 8-18.

Mazza, TO \& Costa, AL. (2014). Análise do absenteísmo de novos pacientes no hospital das Clínicas da Faculdade de Medicina de Ribeirão Preto da Universidade de São Paulo. In Anais do $22^{\circ}$ Simpósio Internacional de Iniciação Científica e Tecnológica da USP, São Paulo, SP.

Medici, AC. (2001). Hospitais universitários: passado, presente e futuro. Revista da Associação Médica Brasileira, 47(2), pp. 149-156.

Ministério da Saúde, Organização Pan-Americana da Saúde. (2013). Introdução à Gestão de Custos em Saúde. Brasília, DF: Autores.

Oleskovicz, M, Oliva, FL, Grisi, CCH, Lima, AC \& Custódio, I. (2014). Overbooking in an outpatient healthcare facility in the Brazilian Unified National Health System. Cadernos de Saúde Pública, 30(5), pp. 1009-1017.

Organização Pan-Americana da Saúde. (2017). Saúde nas Américas+: Resumo do panorama regional e perfil do Brasil. Washington, D.C.: Autor.

Pereira, AS, Shitsuka, DM, Parreira, FJ \& Shitsuka, R. (2018). Metodologia da pesquisa científica. [e-book]. Santa Maria. Ed. UAB/NTE/UFSM. Disponível em: https://repositorio.ufsm.br/bitstream/handle/1/15824/Lic_Computacao_MetodologiaPesquisa-Cientifica.pdf?sequence $=1$.

Pereira-Victorio, CJ, Vázquez-Molinero, A, Lizán-García, M, Gómez-Guardiola, JL \& López-Valcárcel, BG. (2016). Absentismo de pacientes a la consulta externa especializada en un hospital de tercer nivel en España. Medicina General y de Familia, 5(3), p. 83-90.

Piola, SF, Servo, LMS, Sá, EB \& Paiva, AB. (2013). Estruturas de Financiamento e Gasto do Sistema Público de Saúde. In: J. C. Noronha, \& T. R. Pereira (Org). A saúde no Brasil em 2030: estrutura do financiamento e do gasto setorial (pp. 15-70). Rio de Janeiro, RJ: Fiocruz. 
Santos, JS. (2008). Absenteísmo dos usuários em consultas e procedimentos especializados agendados no SUS: um estudo em um município baiano. (Dissertação de Mestrado). Universidade Federal da Bahia, Vitória da Conquista, BA.

Santos, RV, Borgert, A \& Borgert, EA. (2017). Relação entre os custos de um hospital universitário e o reembolso do Sistema Único de Saúde-SUS. In: Anais do XXIV Congresso Brasileiro de Custos. Florianópolis, SC.

Silva, MM, Santanda, NGM, Santos, MC, Cirilo, JD, Barrocas, JD \& Moreira, MC. (2015). Cuidados paliativos na assistência de alta complexidade em oncologia: percepção de enfermeiros. Escola Anna Nery Revista de Enfermagem, 19(3), pp. 460-466.

Siqueira Filha, NT. (2011). Análise de custos em um ambulatório de especialidades médicas: implicações do cancelamento das consultas para pacientes, familiares e sistema de saúde. (Dissertação de Mestrado). Instituição de Medicina Integral Prof. Fernando Figueira, Recife, 2011.

Sodré, F, Littike, D, Drago, LMB \& Perim, MCM. (2013). Empresa Brasileira de Serviços Hospitalares (The Brazilian Company of Hospital Services): a new management model? Serviço Social e Sociedade, (114), pp. 365-380.

Solla, JJSP \& Chioro, A. (2012). Atenção ambulatorial especializada. In: In L. Giovanella, S. Escorel, L. V. C. Lobato, J. C. Noronha, \& A. I. Carvalho (Org.), Políticas e sistema de saúde no Brasil (2 ed., Cap. 17, pp. 547-576) Rio de Janeiro, RJ: Cebes e Fiocruz.

Sousa, AMC. (2014). Universalidade da saúde no Brasil e as contradições da sua negação como direito de todos. Revista Katálysis, 17(2), pp. 227-234.

Stone, CA, Palmer, JH, Saxby, PJ \& Devaraj, VS. (1999). Reducing non-attendance at outpatient clinics. Journal of the Royal Society of Medicine, 92(3), pp. 114-118.

Tristão, FI. (2016). Absenteísmo dos usuários aos serviços de saúde no município de Vila Velha no Estado do Espírito Santo. (Dissertação de Mestrado). Universidade Federal do Espírito Santo, Vila Velha, ES. 
Ugá, MAD, Porto, SM \& Piola, SF. (2012) Financiamento e Alocação de Recursos em Saúde no Brasil. In: L. Giovanella, S. Escorel, L. V. C. Lobato, J. C. Noronha, \& A. I. Carvalho (Org), Políticas e sistema de saúde no Brasil (2 ed., Cap. 13, pp. 395-425) Rio de Janeiro, RJ: Cebes e Fiocruz.

Vieira, FS. (2017). Produção de Informação de custos para a tomada de decisão no sistema único de saúde: uma questão para a política pública. Rio de Janeiro - Brasília: IPEA. Colocar espaço entre uma referência e outra. Lembre que usamos APA.

\section{Porcentagem de contribuição de cada autor no manuscrito}

Fabricio Varoni de Oliveira - 60\%

Reisoli Bender Filho - 25\%

Kelmara Mendes Vieira $-15 \%$ 
Research, Society and Development, v. 9, n. 7, e417974066, 2020

(CC BY 4.0) | ISSN 2525-3409 | DOI: http://dx.doi.org/10.33448/rsd-v9i7.4066

APÊNDICE A - Custo do Ambulatório de Atenção Psicossocial do HUSM entre 2015 e

2017, em R\$ e em \%.

\begin{tabular}{lrrrrrr}
\hline \multicolumn{1}{c}{ Itens de custo } & \multicolumn{1}{c}{ 2015 } & \multicolumn{1}{c}{$\%$} & \multicolumn{1}{c}{2016} & \multicolumn{1}{c}{$\%$} & \multicolumn{1}{c}{2017} & \multicolumn{1}{c}{$\%$} \\
\hline Fol. de pagamento - Médicos & $1.176 .188,70$ & 62,19 & $1.189 .859,52$ & 59,67 & $1.295 .153,31$ & 60,25 \\
Fol. de pagamento - Administrativo & $109.919,08$ & 5,81 & $122.885,05$ & 6,16 & $134.340,79$ & 6,25 \\
Fol. de pagamento - Enfermagem & $93.335,53$ & 4,94 & $100.539,49$ & 5,04 & $124.915,08$ & 5,81 \\
Bolsa de estudo - Residência & $321.436,08$ & 17,00 & $353.311,38$ & 17,72 & $359.686,44$ & 16,73 \\
Recepcionistas & $54.173,55$ & 2,86 & $63.280,75$ & 3,17 & $65.846,09$ & 3,06 \\
Manutenção e suporte em Computadores & $10.318,63$ & 0,55 & $11.566,52$ & 0,58 & $12.551,89$ & 0,58 \\
Manutenção e suporte em Telefonia & $3.283,45$ & 0,17 & $3.574,97$ & 0,18 & $4.535,62$ & 0,21 \\
Custo com ligações telefônicas ${ }^{1}$ & 446,69 & 0,03 & 471,35 & 0,02 & 486,68 & 0,02 \\
Vigilância & $38.159,46$ & 2,02 & $44.624,39$ & 2,24 & $47.442,17$ & 2,21 \\
Radiocomunicadores & 426,81 & 0,02 & 493,82 & 0,02 & 435,53 & 0,02 \\
Monitoramento & $3.235,57$ & 0,17 & $3.743,52$ & 0,19 & $2.458,60$ & 0,11 \\
Serviço de Arquivo & $25.695,89$ & 1,36 & $33.914,18$ & 1,70 & $32.700,17$ & 1,52 \\
Serviço de Limpeza & $28.302,43$ & 1,50 & $34.056,80$ & 1,71 & $37.690,18$ & 1,75 \\
Serviço de Água & $3.218,23$ & 0,17 & $4.419,41$ & 0,22 & $5.160,43$ & 0,24 \\
Energia Elétrica & $23.016,95$ & 1,22 & $27.238,93$ & 1,37 & $26.214,71$ & 1,22 \\
\hline Total & $1.891 .157,06$ & 100,00 & $1.993 .980,07$ & 100,00 & $2.149 .617,69$ & 100,00 \\
\hline
\end{tabular}

Fonte: Resultado da pesquisa. Elaborado pelos autores.

${ }^{1}$ Atualizado pelo Índice de Serviços de Telecomunicações (IST/ANATEL). 